\title{
Involvement of CCDC102A in Artesunate-induced apoptosis in human U937 cells
}

\author{
Ying $\mathrm{Li}^{1}$, ruijie sun ${ }^{1}$, qian wang ${ }^{1}$, ningning $\operatorname{shan}^{1}$, hongzhi $\mathrm{xu}^{1}$, linlin $\mathrm{zhu}^{1}$, and xin liu ${ }^{1}$ \\ ${ }^{1}$ Shandong Provincial Hospital
}

April 28, 2020

\begin{abstract}
Background: To investigate the effects of Artesunate (ART) on cell proliferation inhibition, apoptosis as well as gene expression of leukemic monocytic lymphoma cell line U937, and discuss the underlying mechanism. Methods: U937 cells were divided into groups treated by ART at different concentrations. Inhibiting effects of ART were determined by CCK-8 assay. Illumina Hiseq platform combined with q-PCR technology was applied to select the specific differentially expressed gene (DEG) between ART treated group and control group; Cell apoptosis was investigated by flow cytometry, and the expressions of apoptosis-related proteins were detected by western blot with CCDC102A being interfered. Results: ART possessed inhibitory effect on U937 cells proliferation in a concentration-dependent manner. The cell viability was no longer decreasing after the concentration of ART increased to $50 \mathrm{mg} / \mathrm{L}$. CCDC102A gene was selected as the target gene through DEGs analysis to be interfered, in order to study the effects on U937. After interfered CCDC102A, the proliferation of U937 was inhibited, while the apoptosis was enhanced, the expressions of pro-apoptotic proteins such as Bax, Active-Caspase 3 as well as p53 were increased, and the expression of anti-apoptotic protein Bcl2 was suppressed. Furthermore, the expressions of proteins in PI3K/Akt/mTOR signaling pathway such as AKT, p-AKT and mTOR were inhibited. Conclusion: ART can inhibit growth of U937 cell and induce apoptosis. CCDC102A gene may be involved in the anti-cancer effects of ART through induction apoptosis of U937 cells via up-regulation of Bax/Bcl2 levels and inhibition of Akt-mTOR signaling pathways.
\end{abstract}

\section{Hosted file}

manuscript . doc available at https : //authorea. com/users/313816/articles/444242-involvement-ofccdc102a-in-artesunate-induced-apoptosis-in-human-u937-cells

A

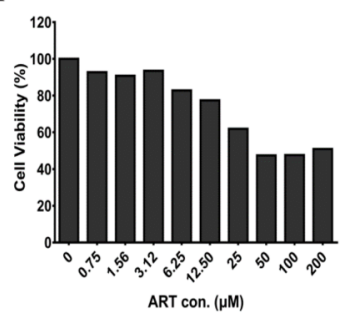

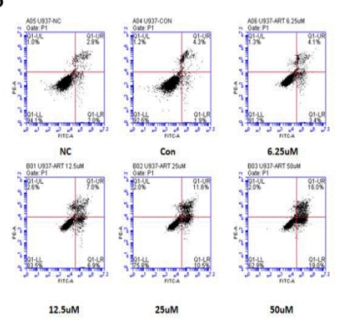

$\mathrm{C}$

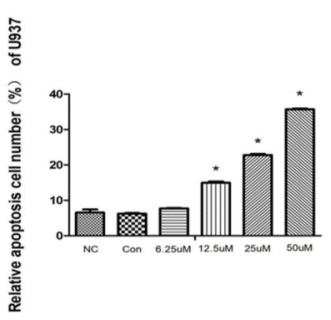


A

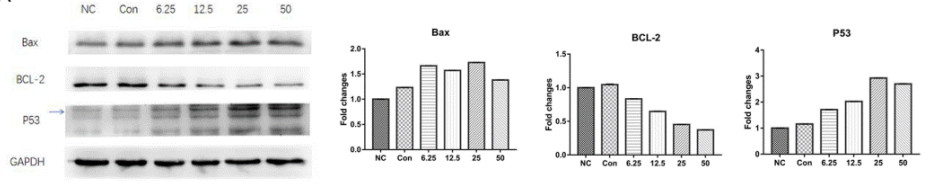

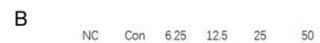

${ }_{\text {Akt }}-\cdots-\cdots$

P-Akt $=-=$

${ }_{\text {MTOR } \rightarrow \text { I D }} \rightarrow \square \square$

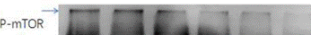

${ }_{\mathrm{GAPDH}}----$

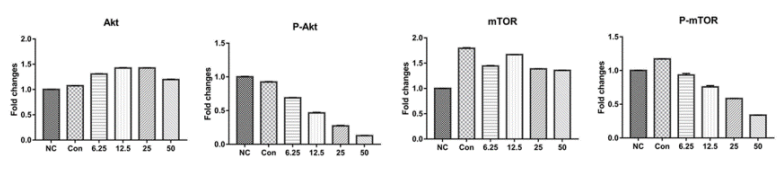

(A)

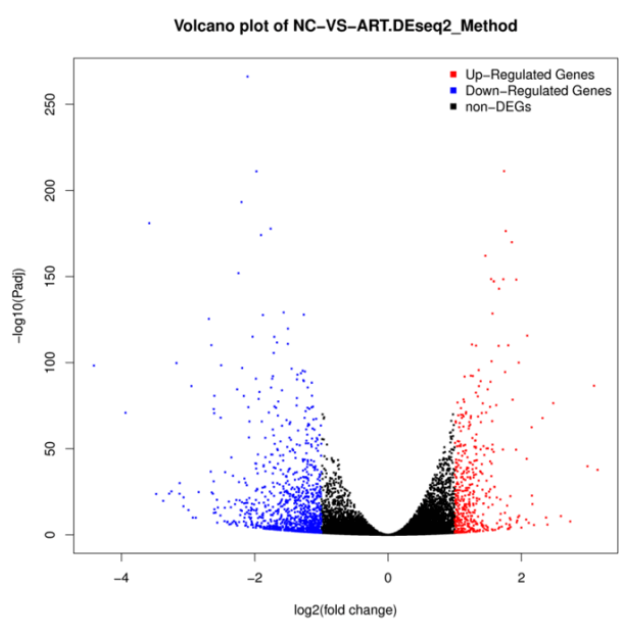

(B)

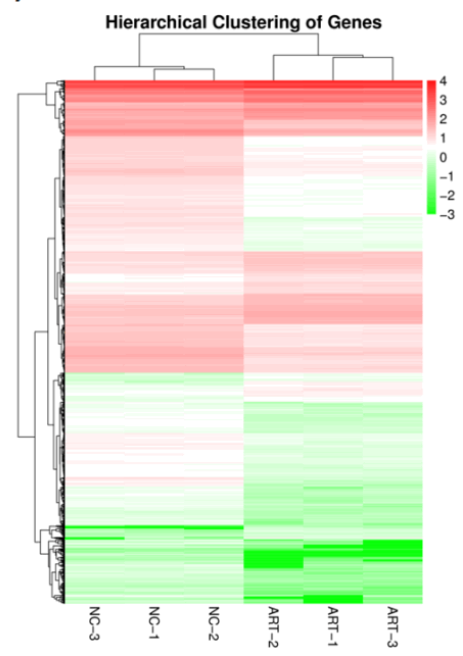

A

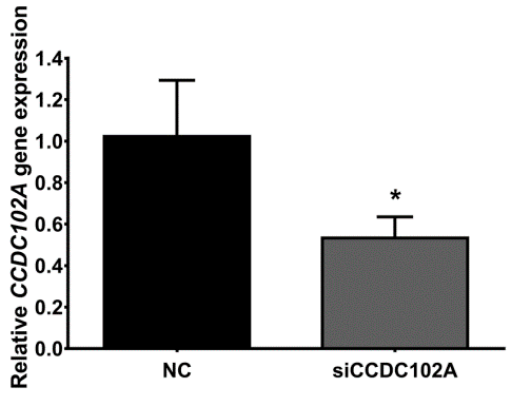

B

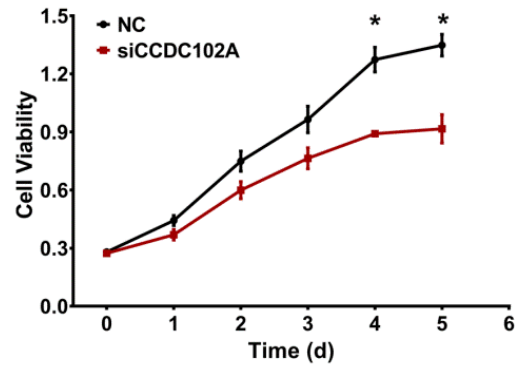


A
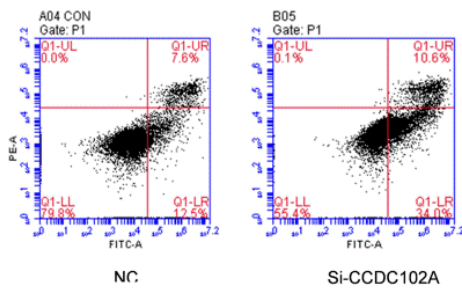

C

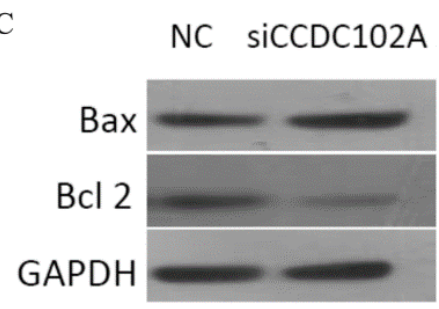

A

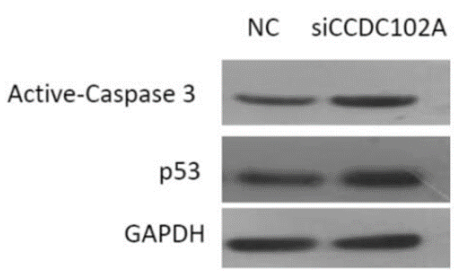

C

NC siCCDC102A D

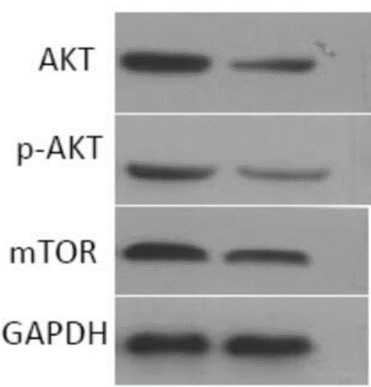

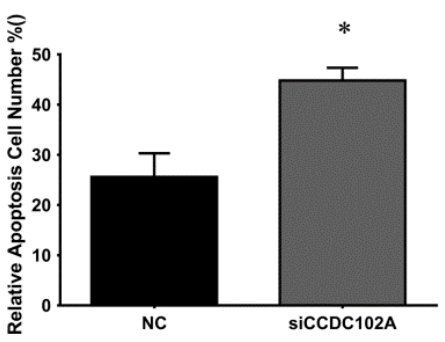

D

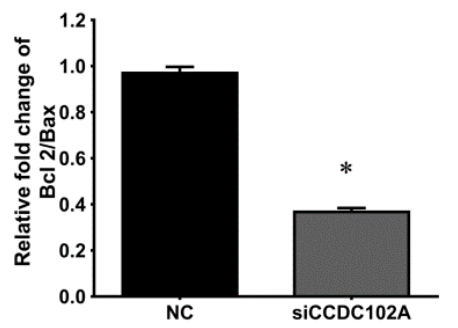

B
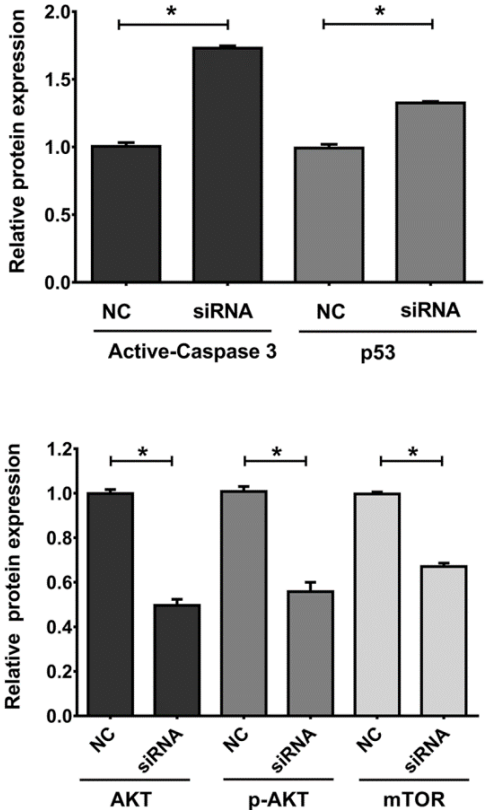


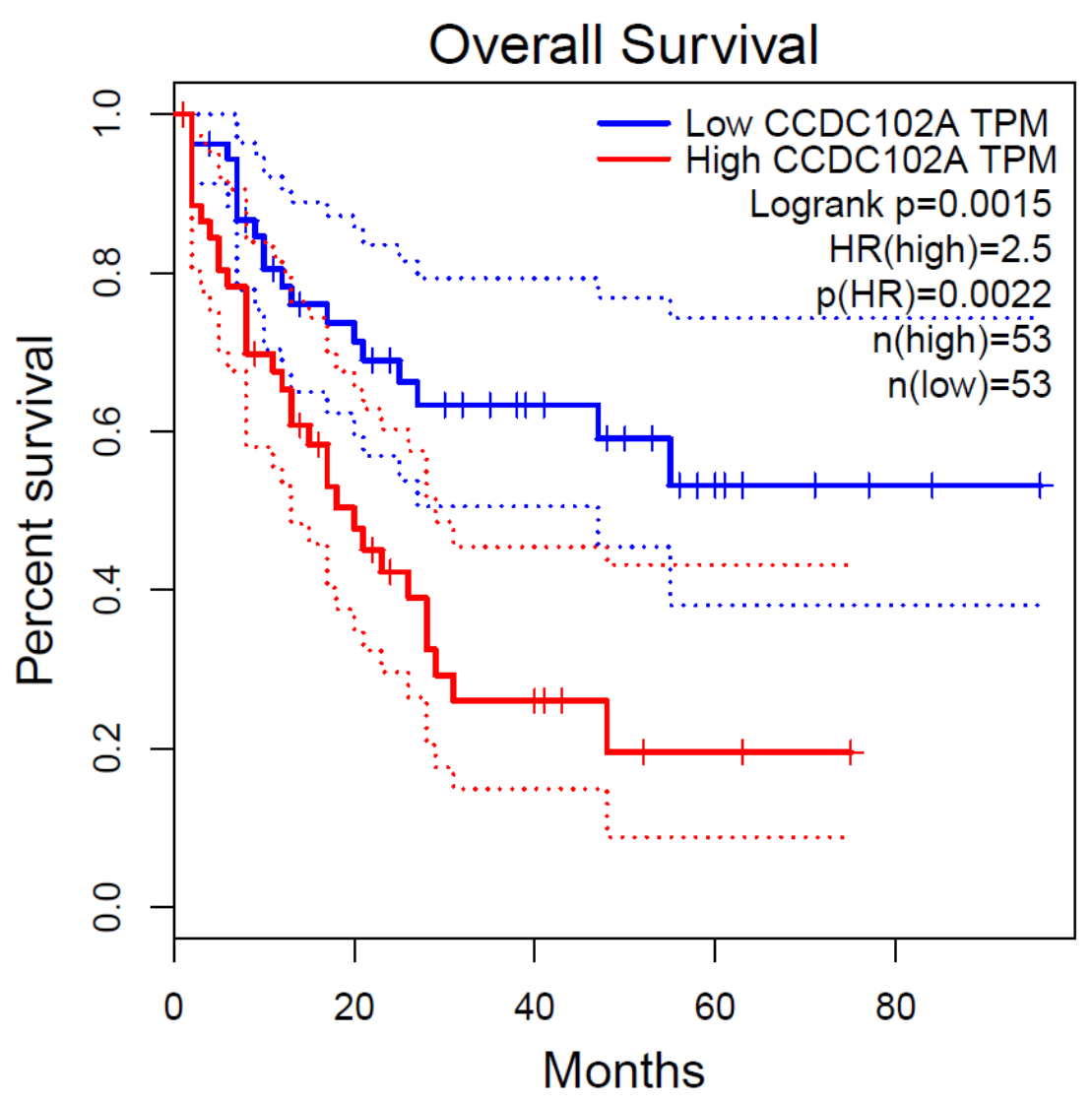

